\title{
AUTHOR INDEX \\ VOLUME 42 (2016)
}

Chan, F.-T., see Yin, J.-H.

Chan, F.-T., see Yin, J.-H.

Chang, C.-W., see Kuan, N.-L.

Chang, C.-Y., see Yang, C.

Chang, H.-K., see Hsu, J. C.-N.

Chang, H.-K., see Hsu, J. C.-N.

Chang, H.-K., see Tsai, F.-Y.

Chang, H.-M., see Tsai, F.-Y.

Chang, H.-W., see Chang, Y.-C.

Chang, H.-W., see Li, W.-T.

Chang, H.-W., see Yang, C.

Chang, H.-Y., Lai, J.-M. and Wang, J.-H., The Minimum Inhibitory Concentration of

Antibiotics Against Nocardia seriolae Isolation from Diseased Fish in Taiwan

Chang, L.-J., see Kao, C.-F.

Chang, L.-W., see Chung, S.-H.

Chang, P.-H., see Chen, I.-W.

Chang, P.-H., see Kao, C.-F.

Chang, S.-C., see Lee, R.-K.

Chang, W.-F., see Yin, J.-H.

Chang, W.-F., see Yin, J.-H.

Chang, Y.-C., Jeng, C.-R., Pang, V. F., Lai, Y.-H., Lien, C.-Y., Wang, P.-J., Guo, C.-C. and Chang, H.-W., Post-Anesthesia Hiatus Hernia in a Bengal Tiger (Panthera tigris tigris)

Chang, Y.-C., see Yang, C.

Chen, H. C., see $\mathrm{Su}$, B.-G.

Chen, H.-W., Hsiao, T.-H. and Wang, C.-H., Detection of Anti-Reticuloendotheliosis Antibody by Enzyme-Linked Immunosorbent Assay Using Envelope Protein Expressed in Baculovirus

Chen, I.-W., Chang, P.-H., Chen, M.-S., Renault, T., Chen, M.-M., Kuo, S.-T. and Cheng, C.-H., Exploring the Chronic Mortality Affecting Abalones in Taiwan: Differentiation of Abalone Herpesvirus-Associated Acute Infection from Chronic Mortality by PCR and In Situ Hybridization and Histopathology

Chen, J.-Y., see Lee, K.-H.

Chen, K.-L., Lin, J.-L., Yu, C.-Y., Su, Y.-C., Wu, C.-P. and Chu, C., Effects of Challenge Methods and Feeding Additives on Antibody Production and Growth Inhibition of the Serum Against Salmonella Enteritidis, S. Gallinarum and S. Pullorum in Chicken Chen, K.-S., see Hsu, J. C.-N.

Chen, K.-S., see $\mathrm{Su}, \mathrm{W} .-\mathrm{L}$.

Chen, M.-M., see Chen, I.-W.

Chen, M.-S., see Chen, I.-W.

Chen, W.-Y., see Chung, S.-H.

Chen, Y.-N., see Su, B.-G.
1 (2016) 27-30

1 (2016) $31-34$

3 (2016) 143-148

4 (2016) 187-193

3 (2016) 177-180

4 (2016) 213-217

3 (2016) 181-186

3 (2016) 181-186

3 (2016) 171-175

1 (2016) 47-52

4 (2016) 187-193

2 (2016) 81-84

1 (2016) 41-46

2 (2016) 53-67

1 (2016) $1-9$

1 (2016) 41-46

1 (2016) 35-39

1 (2016) 27-30

1 (2016) 31-34

3 (2016) 171-175

4 (2016) 187-193

1 (2016) 19-26

3 (2016) 165-170

1 (2016) 1-9

4 (2016) 203-212

3 (2016) 149-157

3 (2016) 177-180

3 (2016) 159-163

1 (2016) 1-9

1 (2016) $1-9$

2 (2016) 53-67

1 (2016) 19-26 
Cheng, C.-C., see $\mathrm{Su}, \mathrm{W} .-\mathrm{L}$.

Cheng, C.-H., see Chen, I.-W.

Cheng, H.-C., see Su, B.-G.

Cheng, I.-C., see Yang, C.

Cheng, T.-L., see Chung, S.-H.

Cheng, Y., Chou, C.-H. and Tsai, H.-J., Gene Expression of Bovine Peripheral Blood

Mononuclear Cells In Vitro Challenged with Mycobacterium bovis

Chi, C.-H., see Lee, K.-H.

Chou, C.-C., see Chung, S.-H.

Chou, C.-H., see Cheng, Y.

Chu, C., see Chen, K.-L.

Chu, C.-Y., see Lee, J.-W.

Chung, S.-H., Chang, L.-W., Cheng, T.-L., Lin, C.-J., Chen, W.-Y. and Chou, C.-C.,

Establishing In-House Reference Intervals for Dogs in Veterinary Clinics

Dizaji, V. M., Kazemi, D. and Rezaei, M., Influence of Platelet Rich Fibrin on

Biomechanical Properties of Primary Sutured Cutaneous Incisional Wounds

Guo, C.-C., see Chang, Y.-C.

Ho, C.-L., see Hsu, J. C.-N.

Hou, F.-H., see Hsu, J. C.-N.

Hsiao, S.-T., see Yin, J.-H.

Hsiao, T.-H., see Chen, H.-W.

Hsu, J. C.-N., Chang, H.-K., Ho, C.-L., Chen, K.-S., Wu, H.-Y., Hsu, T.-H. and Lin, C.-C., Comminuted Fracture of Right Tarsal Bones with Old Callus Formation in a Slaughtered Pig

Hsu, J. C.-N., Lin, C.-C., Hou, F.-H., Chang, H.-K., Hsu, T.-H., Liao, J.-W.

and Lee, W.-C., Case Report: Isoimmune Thrombocytopenic Purpura in Suckling Pigs

Hsu, K.-C., see Lee, K.-H.

Hsu, T.-H., see Hsu, J. C.-N.

Hsu, T.-H., see Hsu, J. C.-N.

Hsu, T.-H., see $\mathrm{Su}, \mathrm{W} .-\mathrm{L}$.

Jea, Y.-S., see Lee, K.-H.

Jeng, C.-R., see Chang, Y.-C.

Jeng, C.-R., see Li, W.-T.

Jeng, C.-R., see Yang, C.

Kao, C.-F., Chang, L.-J., Lee, Y. and Chang, P.-H., Case Report: A Chronic B-Cell

Leukemia in a Eurasian Otter (Lutra lutra)

Kao, J.-P., see Tsai, F.-Y.

Kazemi, D., see Dizaji, V. M.

Ke, G.-M., see Lee, J.-W.

Kuan, N.-L., Chang, C.-W., Lee, C.-A. and Yeh, K.-S., Extended-Spectrum Beta-

Lactamase-Producing Escherichia Coli and Klebsiella pneumoniae Isolates from the Urine

of Dogs and Cats Suspected of Urinary Tract Infection in a Veterinary Teaching Hospital

Kuo, S.-T., see Chen, I.-W.

Lai, J.-M., see Chang, H.-Y.

Lai, Y.-H., see Chang, Y.-C.

Lee, C.-A., see Kuan, N.-L.

Lee, J.-W., Lin, Y.-M., Liu, C.-H., Ke, G.-M. and Chu, C.-Y., Passive and Protective

Immunity in Ducklings Elicited by a Parvovirus Subunit Vaccine with CpG Adjuvant

Lee, K.-H., Hsu, K.-C., Wang, Y.-S., Yeh, C.-C., Chen, J.-Y., Wang, S.-H., Jea, Y.-S. and

Chi, C.-H., Effects of Taraxacum mongolicum Extract on Lipopolysaccharide-Induced

Nitric Oxide and Cytokines Production by Bovine Peripheral Blood Mononuclear Cells
3 (2016) 159-163

1 (2016) $1-9$

1 (2016) $19-26$

4 (2016) $187-193$

2 (2016) 53-67

4 (2016) 195-201

4 (2016) 203-212

2 (2016) 53-67

4 (2016) 195-201

3 (2016) 149-157

2 (2016) 75-80

2 (2016) 53-67

2 (2016) 69-74

3 (2016) 171-175

3 (2016) 177-180

4 (2016) 213-217

1 (2016) 31-34

3 (2016) 165-170

3 (2016) 177-180

4 (2016) 213-217

4 (2016) 203-212

4 (2016) 213-217

3 (2016) 177-180

3 (2016) 159-163

4 (2016) 203-212

3 (2016) 171-175

1 (2016) 47-52

4 (2016) 187-193

1 (2016) 41-46

3 (2016) 181-186

2 (2016) 69-74

2 (2016) 75-80

3 (2016) 143-148

1 (2016) 1-9

2 (2016) 81-84

3 (2016) 171-175

3 (2016) 143-148

2 (2016) 75-80

4 (2016) 203-212 
Lee, R.-K., Tsai, Y.-L., Wang, H.-J., Lin, C.-C., Chang, S.-C. and Liao, J.-W., Case Report: Hemangiosarcoma of the Mesentery in a Ferret (Mustela putorus furo)
1 (2016) 35-39
4 (2016) 213-217

Lee, W.-C., see Hsu, J. C.-N.

Lee, W.-M., see $\mathrm{Su}, \mathrm{W} .-\mathrm{L}$.

Lee, Y., see Kao, C.-F.

Li, W.-T., Jeng, C.-R., Liu, C.-H., Pang, V. F., Wang, C.-H., Wang, P.-J. and Chang, H.-W., Morphological and Immunohistochemical Characterization of a Rhabdomyosarcoma with Systemic Metastasis in an Avian Leukosis Virus (ALV) Infected Chicken

Liao, J.-W., see Hsu, J. C.-N.

Liao, J.-W., see Lee, R.-K.

Liao, J.-W., see Tsai, F.-Y.

Liao, J.-W., see Yin, J.-H.

Liao, J.-W., see Yin, J.-H.

Lien, C.-Y., see Chang, Y.-C.

Lin, C.-C., see Hsu, J. C.-N.

Lin, C.-C., see Hsu, J. C.-N.

Lin, C.-C., see Lee, R.-K.

Lin, C.-J., see Chung, S.-H.

Lin, J.-L., see Chen, K.-L.

Lin, Y.-M., see Lee, J.-W.

Liu, C.-H., see Lee, J.-W.

Liu, C.-H., see Li, W.-T.

Mao, C.-J., see Yin, J.-H.

Pang, V. F., see Chang, Y.-C.

Pang, V. F., see Li, W.-T.

Pang, V. F., see Yang, C.

Proceedings of the Joint Fall Conference and Zoonosis Workshop of the Chinese Society of Veterinary Science and the Taiwan Association of Veterinary Science and Animal Husbandry (CSVS/TAVSAH)

Proceedings of the 2016 Joint Spring Conference of the Chinese Society of Veterinary Science and the Taiwan Association of Veterinary Science and Animal Husbandry

(CSVS/TAVSAH)

Renault, T., see Chen, I.-W.

Rezaei, M., see Dizaji, V. M.

Shia, W.-Y., see Su, W.-L.

Shih, H.-Y., Yu, J.-F. and Wang, L.-C., Stereotypic Behaviors in Bears

Su, B.-G., Chen, H. C., Cheng, H.-C. and Chen, Y.-N., Detection of Bat Coronavirus and Specific Antibodies in Chestnut Bat (Scotophilus kuhlii) Population in Central Taiwan

Su, W.-L., Cheng, C.-C., Shia, W.-Y., Hsu, T.-H., Chen, K.-S., Wang, H.-C. and Lee, W.-M., Effect of Trivalent Chromium on Growth Hormone and Insulin-Like Growth Factor-I in Ovariohysterectomized Rats

$\mathrm{Su}$, Y.-C., see Chen, K.-L.

Tsai, F.-Y., Chang, H.-M., Chang, H.-K., Kao, J.-P. and Liao, J.-W., Case Report: Endometrial Stromal Sarcoma and Liposarcoma in an African Hedgehog (Atelerix albiventris)

Tsai, H.-J., see Cheng, Y.

Tsai, P.-S., see Yang, C.

Tsai, Y.-L., see Lee, R.-K.

Tsai, Y.-L., see Yin, J.-H.

Wang, C.-H., see Chen, H.-W.

1 (2016) 47-52

4 (2016) $213-217$

1 (2016) 35-39

3 (2016) 181-186

1 (2016) 27-30

1 (2016) 31-34

3 (2016) 171-175

4 (2016) 213-217

3 (2016) 177-180

1 (2016) 35-39

2 (2016) 53-67

3 (2016) 149-157

2 (2016) 75-80

2 (2016) 75-80

1 (2016) 47-52

1 (2016) 27-30

3 (2016) 171-175

1 (2016) 47-52

4 (2016) 187-193

4 (2016) 219-258

2 (2016) 85-142

1 (2016) 1-9

2 (2016) 69-74

3 (2016) 159-163

1 (2016) 11-17

1 (2016) 19-26

3 (2016) 159-163

3 (2016) 149-157

3 (2016) 181-186

4 (2016) 195-201

4 (2016) 187-193

1 (2016) 35-39

1 (2016) 31-34

3 (2016) 165-170 
Wang, C.-H., see Li, W.-T.

Wang, H.-C., see $\mathrm{Su}, \mathrm{W} .-\mathrm{L}$.

Wang, H.-J., see Lee, R.-K.

Wang, J.-H., see Chang, H.-Y.

Wang, L.-C., see Shih, H.-Y.

Wang, P.-J., see Chang, Y.-C.

Wang, P.-J., see Li, W.-T.

Wang, S.-H., see Lee, K.-H.

Wang, Y.-S., see Lee, K.-H.

Wu, C.-P., see Chen, K.-L.

Wu, H.-Y., see Hsu, J. C.-N.

Yang, C., Chang, Y.-C., Chang, C.-Y., Tsai, P.-S., Jeng, C.-R., Pang, V. F., Cheng, I.-C. and Chang, H.-W., Identification of a Highly Transfectable Cell Line Permissive to Porcine Epidemic Diarrhea Virus Infection and Replication

Yeh, C.-C., see Lee, K.-H.

Yeh, K.-S., see Kuan, N.-L.

Yen, H.-C., see Yin, J.-H.

Yin, J.-H., Tsai, Y.-L., Chang, W.-F., Hsiao, S.-T., Chan, F.-T. and Liao, J.-W., Case Report: Osteoporosis and Osteomalacia with Multiple Bone Fractures in a Auntiacus reevesi

Yin, J.-H., Yen, H.-C., Chang, W.-F., Mao, C.-J., Chan, F.-T. and Liao, J.-W., Case Report: Suspected Botulism in a Black-Faced Spoonbill (Platalea minor)

Yu, C.-Y., see Chen, K.-L.

Yu, J.-F., see Shih, H.-Y.
1 (2016) 47-52

3 (2016) 159-163

1 (2016) 35-39

2 (2016) 81-84

1 (2016) 11-17

3 (2016) 171-175

1 (2016) 47-52

4 (2016) 203-212

4 (2016) 203-212

3 (2016) 149-157

3 (2016) 177-180

4 (2016) 187-193

4 (2016) 203-212

3 (2016) 143-148

1 (2016) 27-30

1 (2016) 31-34

1 (2016) 27-30

3 (2016) 149-157

1 (2016) 11-17 